\title{
Relaciones Públicas y el desarrollo sostenible en duda en los trabajos académicos franceses ${ }^{1}$
}

Recibido: 14 de febrero de 2011

Aceptado: 08 de agosto de 2011

Publicado: 31 de octubre de 2011
Arlette Bouzon arlette.bouzon@iut-tlse3.fr Joëlle Devillard joelle.devillard@iut-tlse3.fr (Universidad de Toulouse - Francia)

Resumen: Las necesidades de informar y convencer son milenarias y son parte de la vida social. Debido a la naturaleza misma de sus actividades en el ámbito económico y social (el Know-How ya no es posible sin el Make-Known) la organización (generalmente las compañías, asociaciones, el sector público) se ha convertido por sí misma en un agente de comunicación.

En este contexto difícil y de incertidumbre, la comunicación parece ser esencial en el proceso que implica el desarrollo sostenible (DS), principalmente cuando se evalúan los informes correspondientes. Parece ser un asunto especialmente delicado que presenta inherentemente un riesgo para la organización. ¿Debería uno permanecer en silencio? ¿O debería hablar? Y si es así, ¿cómo debería plantear las cosas y qué tan lejos debería revelarse el nivel de incertidumbre?

La comunicación sobre DS constituye un problema emergente cuyo significado va mucho más allá de la usual especulación relacionada a la comunicación en momentos de crisis.

En el presente artículo, se revisan los trabajos que abarcan la comunicación organizacional, enfocados en los orígenes conceptuales de los términos "comunicación" y "organización". De esta forma, se presenta un panorama de los trabajos franceses sobre estos tópicos, subrayándose un interés especial en las bases epistemológicas subyacentes.

Palabras clave: Relaciones Públicas, desarrollo sostenible, agente de comunicación, comunicación organizacional en Francia.

1.- Artículo escrito originalmente en inglés. Traducido al español por Jessica Chipoco Fraguela. 
Abstract: The needs to inform and convince are millenniums and part of any organized life. Due to the very nature of its activities in the economic and social sphere, and because the "Know-How" is no longer possible without the "Make-Known", the organization (generally companies, associations, the public sector) has turned itself into a communication agent.

In this difficult and uncertain present context, communication appears to be essential in the process of gradually becoming better aware of sustainable development, mainly when it comes to how the corresponding reports are devised. It seems to be an especially delicate matter inherently presenting a risk for the organization. Should one remain silent? Or should one speak out? And if so, how should one put things and how far should the level of uncertainty be revealed?

So, communication around Sustainable Development constitutes an emerging problem area whose significance goes well beyond the usual speculation related to crisis communication which latter only addresses one of its facets.

In the present article, works covering organizational communication are reviewed, focusing on the existing scientific literature on the subject. We shall thus be led to re-direct our attention to the conceptual and theoretical origins of the communication and organization couple, taking a special interest in the underlying epistemological foundations.

Key words: Public Relations, Sustainable Development, Communication Agent, Corporate Communications in France.

\section{Introducción}

Las organizaciones (compañías, asociaciones, sector público) se han convertido en activos y dinámicos agentes de comunicación. Ser conocidos entre un público dado, atraer y mantener a los compañeros de trabajo; informar a los accionistas, obtener oportunidades en la banca, ganarse el favor de las autoridades y asociaciones locales impone estos diversos requerimientos que han de satisfacerse. Hablar, ser asertivo e intercambiar ideas es lo más importante porque todos estos actos y características pueden ser considerados como mensajes (nombre, producto, empaque, comportamiento del personal) y también porque una parte importante de la comunicación (entablada de manera informal dentro de la organización o en el "boca a boca" fuera de ella) no se emite de una manera deliberada.

Supone que cada medio de comunicación disponible se defina precisamente por los comunicadores involucrados. Por ello, en los trabajos académicos franceses, las Relaciones Públicas corresponden a un conjunto de técnicas de comunicación utilizadas por una organización (pública o privada) para establecer, mantener o desarrollar relaciones confiables con un público dado, mediante un intermediario (la relación es "triangular"). Dirigida al personal que trabaja dentro de la organización, las RR. PP. a nivel interno tratan de asegurar y desarrollar una atmósfera de confianza, para fortalecer la cohesión entre las diversas partes constituyentes, para reafirmar una cultura corporativa y para movilizar a los empleados. Del mismo modo, a nivel externo, las RR. PP. trabajan para 
crear o desarrollar un capital de simpatía y fortalecer una imagen positiva. Sus acciones se transforman principalmente en operaciones de auspicio, eventos culturales, deportes $\mathrm{u}$ operaciones caritativas, invitaciones a fiestas u operaciones exitosas y, sobre todo, a estrechar relaciones con los medios, buscando obtener informes positivos de periodistas a favor del anunciante en cuestión.

En cambio, la Publicidad buscará actuar más sobre el comportamiento de los individuos involucrados que por el objetivo al que se busca llegar. Será definida como un proceso de comunicación dirigido, que emana de una organización con la visión de comunicar a un público dado un elemento de información (una oferta existente o hecho) mediante diversos espacios comprados en los medios: prensa, radio, televisión, cine, avisos, Internet, entre otros. Debemos subrayar en este punto que la Publicidad ha evolucionado mucho, principalmente debido a los panoramas legales y éticos que han sido modificados.

Hoy en día, la creciente interdependencia de los mercados, el desarrollo de productos estandarizados, el surgimiento de los medios internacionales y la reducción de los presupuestos de comunicación durante algunos años, han conducido a una evolución en los requerimientos del desempeño comunicacional, como lo muestran los numerosos debates sobre el "neuro-marketing" entre los profesionales franceses.

\section{Metodología de trabajo}

Adoptamos un enfoque comparativo-descriptivo que nos permite evaluar, con una mirada panorámica estructurada, la importancia de los trabajos críticos entre las investigaciones recientes. En ese sentido, se revisaron las comunicaciones de las conferencias sobre la comunicación organizacional desde el año 2000 y los contenidos de Communication et organisation, una publicación especializada en este campo. De hecho, el artículo científico encarna la legitimidad de una producción científica evaluada por los expertos en este campo, con revisiones anónimas entre pares. Además, constituye una fuente de conocimientos constantemente actualizada y un medio privilegiado de información sobre las actuales investigaciones.

Para estudiar la producción seleccionada en habla francesa, se utilizó una cuadrícula de clasificación. Ante la ausencia de trabajos franceses sobre el tema, se hace referencia a las distintas categorizaciones norteamericanas existentes en las ciencias humanas y sociales (Alvesson, Deetz, 1996; Deetz, 1996; Hardy y Clegg, 1997; Giroux, Marroquin, 2005; Koenig, 2006; Putnam, 1982), entre ellas la de Burrell y Morgan (1979). Muy criticada en su momento, esta matriz se basa en dos hipótesis: la naturaleza de las ciencias sociales (objetiva o subjetiva) y la de la ciencia (cambio radical o regulación). Así, se obtuvieron cuatro paradigmas sociológicos ("funcionalista", "interpretativo", "radical humanista" y "radical estructuralista").

Asimismo, se eligió una versión mejorada de dicha matriz (Giroux y Demers, 1998) quienes asocian los dos últimos paradigmas (radicales) bajo la categoría de "paradigma crítico", evitándose, de esa manera, algunas limitaciones (por ejemplo, la imposibilidad de diálogo 
entre los investigadores). También refleja las evoluciones teóricas recientes, seleccionando los paradigmas epistemológicos identificados ("funcionalista", "interpretativista" y "crítico") y que parecen ser compartidos por un cierto número de investigadores en nuestro campo en ambos lados del Atlántico (Fauré y Bouzon, 2010). Así, nos limitamos voluntariamente a estos paradigmas predominantes sin considerar a los menores. Para analizar los contenidos de los textos seleccionados, adoptamos la cuadrícula metodológica elaborada por Morillon, Aldebert y Szafrajzen (2010), cuya principal ventaja es la de organizar los diversos textos desde el punto de vista epistemológico reclamado por los autores o deducido de las palabras claves que ellos mismos han seleccionado. Además, nos parecieron reveladores los vínculos que los investigadores tratan de tejer con la comunidad científica. De esta forma, se pueden obtener algunas conclusiones sobre los trabajos en comunicación organizacional.

No obstante, debemos comprender cómo las estrategias de comunicación para el DS y la Responsabilidad Social Empresarial (RSE) son entendidas por los actores, desde sus diversas formas de representación.

Durante algunos años, hemos estado realizando una encuesta continua a los procesos de diseño en una serie de empresas pertenecientes al sector turismo y transporte. Hasta ahora hemos desarrollado una metodología que combina la observación directa y las entrevistas con los diseñadores. Del mismo modo, las encuestas nos han permitido identificar mejor sus prácticas diarias y comprender sus representaciones sociales. Sin embargo, solamente diez de los entrevistados estuvieron preparados para responder preguntas referidas al DS y la RSE.

En la tradición de los trabajos de inspiración interaccionista y etnometodológica, queríamos basar nuestro enfoque amplio en el proceso de diseño de la comunicación en entrevistas y en el estudio de escritos que combinan gran cantidad de técnicas de recolección de datos (a fin de registrar los prejuicios inherentes en cada uno de ellos) y así compensar y confrontar los resultados empíricos con cada una de las teorías planteadas. Sin embargo, los problemas de confidencialidad demostraron ser prohibitivos, debido probablemente al periodo actual de la severa crisis económica que afecta al país.

En efecto, más allá de la dificultad que implica el acceso a la empresa y a sus actores, nuestra posición como investigadores siempre es una cuestión delicada, con el riesgo considerable de confundir la posición de la investigación con la de la consultoría, a medida que nuestra investigación se relaciona con la información estratégica sobre un campo sensible. Se nos pidió encarecidamente no revelar información alguna que probablemente pudiera hacer que se reconocieran las organizaciones contactadas y que utilicemos solamente la información de naturaleza genérica. Dado que los profesionales involucrados no tenían una vocación para ser entrevistados, explicar su trabajo o brindar información sobre sí mismos (Dubet, 1994), la labor de recolectar datos relevantes resultó un proceso delicado de negociación y, como otros investigadores ya han observado, "las publicaciones sobre lo que los actores piensan son poco comunes; ya que, incluso cuando se obtiene el derecho de escuchar y observar, solamente se escucha lo que se ha acordado escuchar y ver las actividades elegidas por la jerarquía" (Scardigli, 2001). 
En el análisis final, nuestra investigación demuestra necesariamente ser parcial e incompleta, incluso cuando se hizo un intento de basarse en el enfoque comparativo (entre los actores o entre los distintos negocios), con una visión de corroborar los resultados, identificando las diferencias y las similitudes; distinguiendo las categorías o incluso las características comunes (Geertz, 1986).

En el presente artículo, privilegiamos las referencias científicas existentes y los trabajos que probablemente alimentarían nuestra investigación sobre la comunicación y luego continuamos confrontándolos con los resultados de dicha encuesta parcial e incompleta en el campo.

\section{Comunicación y organización}

Regresemos ahora a los orígenes de esta asociación entre la comunicación y la organización, remarcando los trabajos más importantes y substanciales en la investigación francesa contemporánea.

Todas las organizaciones necesitan tener a su disposición información útil y confiable de diversas fuentes, tanto internas como externas y de manera permanente, con la visión de dar forma a las decisiones estratégicas, al desarrollo del conocimiento y a las habilidades del personal; la preservación del patrimonio y, finalmente, la misma supervivencia de la entidad en cuestión. Debido a ello, los problemas comunicacionales relacionados con la organización son múltiples.

En la teoría de la organización (Rojot, 2005), la comunicación (un término polisémico) resulta ser una "palabra baúl" que aparece con frecuencia en los escritos de muchas investigaciones, adquieriendo los alcances de otros términos tales como "orden", "control", "persuasión", "propaganda", "procesamiento de datos", “negociación”, “coordinación”, “interacción” y "retroacción”.

\subsection{Trabajos académicos franceses sobre la comunicación organizacional: una pluralidad de concepciones}

El estudio sobre la comunicación organizacional constituye un fenómeno de origen anglosajón, principalmente americano, que surgió poco después del ingreso de los Estados Unidos en la Segunda Guerra Mundial. Los años 1942-1947 comprenden un periodo de gestación (Redding, 1985) durante los cuales el término “comunicación” apareció de manera cada vez más creciente en una serie de publicaciones especializadas. Fue tan solo en la década del sesenta que la "comunicación organizacional" adoptó una marcada tendencia a confiar en los datos empíricos-objetivos y, de esta manera, adquirir un estatus científico fuertemente asertivo. Además, Redding considera que 1967 fue el año en que se aceptó oficialmente la expresión "comunicación organizacional” como una disciplina emergente $^{2}$, acompañada por una bibliografía especializada sustancial (Voos, 1967).

2.- En dicho año se organizó la primera conferencia sobre Comunicación Organizacional. 
Más adelante, Lee Thayer (1968) publicó un trabajo titulado Communication and Communication Systems ("Comunicación y Sistemas de Comunicación") el cual, derivando su inspiración principalmente de las formulaciones de la "teoría de los sistemas", fue considerado como un tratado teórico exhaustivo sobre la comunicación en las organizaciones. La dupla "comunicación-organización” ahora parecía estar claramente identificada y florecería con el tiempo. Los investigadores Simon y Weick consideraron que "sin comunicación, no puede haber organización" (Euske y Roberts, 1987).

\subsection{Un campo de investigación reciente}

En Francia, la "comunicación organizacional" constituye un tema central de investigación para un creciente número de investigadores de las Ciencias de la Información y de la Comunicación (CIC), una disciplina científica que fue creada institucionalmente a inicios de 1970 (Boure, 2002), que asocia la "información” y la "comunicación”, a diferencia de los trabajos estadounidenses que se citaron previamente. Esta evolución, en parte, está relacionada con la iniciativa tomada por los miembros de la Sociedad Francesa de Ciencias de la Información y la Comunicación (SFSIC) para establecer un estudio y un grupo de investigación sobre la comunicación dentro de las organizaciones, denominado "Org \& Co" (1994).

Desde su creación, este ente ha brindado un escenario para el debate en el que las diversas escuelas de pensamiento pueden confrontar sus problemas, sus metodologías y sus resultados en el contexto francés. De esta manera, las diversas reuniones, talleres, discusiones en mesas redondas y conferencias han conducido a intercambios fructíferos y han brindado a los participantes de diversos orígenes una oportunidad para confrontar sus enfoques y reunir sus recursos conceptuales y métodos de investigación.

Se abarcaron muchos asuntos en un campo de la ciencia que está acentuando, cada vez más, una diferencia distintiva con los enfoques funcionales y/o gerenciales. Estos se ocupan tanto del contenido, del lugar de la información (y su procesamiento) y de los métodos para los actos de comunicación y el papel que estos últimos desempeñan en las situaciones laborales. Sin embargo, también se pueden relacionar con las políticas de comunicación y sus efectos (incluyendo las Relaciones Públicas, la Publicidad, entre otros), los recursos implementados, los procesos de comunicación entre los actores y las interacciones dentro de las organizaciones y los fenómenos cognitivos y sociales adjuntos a ellos.

Sus problemas fundamentales han enfrentado un perfil más agudo y se han enriquecido durante los últimos años, especialmente mediante la investigación sobre los modelos explicativos multidimensionales contra unos antecedentes de críticas para trascender la normativa dominante y los paradigmas proposicionales. De esta manera, la comunicación organizacional es considerada cada vez más un proceso complejo en el que el significado de los mensajes no es una interacción previa dada, sino una construcción en una situación marcada culturalmente que depende de la manera en que se despliega en el tiempo y en el espacio. Siendo a la vez estructurada y estructuradora, ya no se reduce solamente a una sola perspectiva instrumental, en el que el trabajo cognitivo del actor se subestima, sino que resalta el desempeño de los individuos en sus situaciones correspondientes. La organización y la comunicación se construyen de manera independiente: constituyen realidades emergentes que forman una pareja inseparable que tan solo se puede asir mediante un enfoque interdisciplinario. 
Frente a estos antecedentes, un gran número de investigadores se ha dedicado durante varios años a estudiar las estructuras de proyectos que se encuentran, con mayor frecuencia, en las organizaciones. El proyecto como una situación incierta (orientada hacia el futuro) nos hace una señal para reflexionar los procedimientos para la cooperación entre los actores sociales cuyos roles e intereses divergen, dentro de un espacio restringido y en una duración limitada. Pero, ¿cómo se las arreglan estos individuos con sus distintas habilidades y calificaciones para cooperar dentro de un proyecto? Las acciones de los actores parecen verse influenciadas por su representación de la situación.

La acción colectiva implica entonces una representación colectiva o, al menos, un mínimo de consistencia entre las representaciones presentes. Como cualquier grupo organizado, el proyecto es un constructo social que solamente puede existir y sobrevivir si se las arregla para integrar las estrategias divergentes de sus miembros en una producción colectiva. En esta situación, el objeto del proceso y el proceso mismo se forman mediante una influencia mutua.

En tales procesos, aquellos involucrados no pueden delimitar fácilmente sus contribuciones y deben orientar sus actividades en relación a cómo evoluciona el proyecto o las actividades de los demás actores. ¿Cuáles son los lazos que los actores mantienen entre sí y cómo interactúan? El proyecto solamente se puede justificar a los ojos de sus iniciadores si su capacidad de producción cognitiva excede la de los miembros considerados aisladamente. ¿Cómo entonces se “distribuyen" las actividades, se conducen simultáneamente entre distintos actores y movilizan lenguajes y herramientas que son específicos de una habilidad y actividades durante las cuales las etapas de razonamiento se comparten entre los distintos socios asignados? Como la innovación resulta cada vez más de las múltiples actividades, los actores son forzados a ir más allá de su especialización para recombinar sus conocimientos en dominios "híbridos", moviéndose del centro hacia las afueras de sus habilidades y trasgrediendo los límites disciplinarios hacia los especialistas en otras habilidades.

Los trabajos que se realizan actualmente reúnen todos los tipos de conocimientos y se alimentan de múltiples experimentos metodológicos, según los objetos estudiados (contenido, situación, proceso) y la meta final (describir, comprender, intervenir) de la investigación. Los trabajos correspondientes son cubiertos por muchos estudios empíricos que abordan el contenido y/o procedimientos para los actos de comunicación, sus efectos, su papel en las situaciones de trabajo o la construcción discursiva del contexto en particular.

Sin embargo, en este marco, además de los objetos y conceptos específicos de CIC, los investigadores implicados constantemente se ven confrontados por preguntas relacionadas a los métodos de investigación en un contexto en el que sus opciones conceptuales ${ }^{3}$ tienen que acomodarse a las limitaciones de la investigación en el campo. Esto se debe a que realizar una investigación sobre la organización estructurada no es una cuestión fácil (Delcambre, 2000). Además de las dificultades para obtener el acceso a la empresa y a todos

3.- De esta manera, las publicaciones de Yves Winkin y los trabajos de la Escuela de Palo Alto, señalan que "cualquier comunicación muestra dos aspectos: el contenido y la relación, de tal manera que esta última incluye a la primera". Esta idea han sido ampliada y revisada por otros autores. Bougnoux (1997), en particular, realiza una distinción fecunda proponiendo hacer una sustitución y reemplazar el contenido por "información” y relación por “comunicación", una distinción adoptada implícitamente por muchos investigadores sobre la comunicación organizacional. 
los actores implicados, la postura del investigador es delicada, con un riesgo considerable de confundir la actitud de la investigación con la de la consultoría.

En esta situación, las presiones se sienten vivamente y el peligro es real. Además, dado que los protagonistas sociales no tienen la vocación para ser entrevistados (ni para dar cuentas sobre sí mismos ni tampoco para dar información sobre ellos mismos) recopilar datos relevantes resulta lo que a menudo se convierte en un proceso delicado de negociación. Un gran número de publicaciones sugieren cómo enfrentar el problema, considerando justamente que ningún trabajo en las Ciencias Humanas y Sociales (CHS) puede prescindir de la necesidad de un debate en cuanto a las herramientas empíricas utilizadas.

\subsection{Extensión y fragmentación de los trabajos}

Dos rasgos caracterizan a los estudios de comunicación organizacional contemporánea: la "extensión" y la "fragmentación".

La rápida extensión de estas investigaciones, como se corrobora por el número de investigadores que se encuentran ahora en lista, surgió sorpresivamente con el primer trabajo colectivo del grupo Org \& Co (Le Moenne, 1998), el cual tenía como objetivo reunir a los especialistas dispersos en la materia y buscar el reconocimiento del campo de la comunicación organizacional por derecho propio. Sin embargo, el tema de la comunicación organizacional es aún más atractivo para los investigadores, ya que, en el contexto actual de la globalización, las organizaciones están adoptando cada vez más las reglas operativas que los liberan del marco de trabajo convencional y los plazos de tiempo que modifican el lugar y el papel de la comunicación.

Esto es paralelo a un fenómeno de fragmentación ${ }^{4}$ que resulta de la desintegración de los campos de especialización y la influencia de los paradigmas en competencia. Estos procesos se muestran en las referencias bibliográficas movilizadas por los investigadores en sus trabajos, como reveladores de los vínculos que tejen con la comunidad científica. Somos testigos de un florecimiento del número de referencias, las cuales están relacionadas con aquellas de disciplinas distintas. Con esta doble evolución, la comunicación organizacional está llegando a un acuerdo con una pluralidad de explicaciones que si bien no perjudican su validez epistemológica, sí crean una cierta "marginalidad” (Dogan, Pahre, 1991) que demuestra ser creativa y que brinda una fuente de innovación ${ }^{5}$. En efecto, el mosaico de trabajos existentes no carece de consistencia. La mayoría de trabajos encajan en el cuestionamiento más general relacionado al lugar y el papel de la comunicación organizacional que, considerada como una forma colectiva de inteligencia (en el significado que le dieron Lacoste y Grosjean, 1999), todavía sigue siendo un enigma incluso cuando ha generado una variedad de modelos y ya ha movilizado a un gran número de investigadores de diversas disciplinas.

Mediante su apertura, los enfoques generalistas y específicos en torno a la comunicación organizacional ofrecen al investigador un amplio espectro de preguntas que difieren según sus temas (estudiando un contenido, analizando un proceso) y varían con respecto a su

4.- Tendencia claramente notable en la elección de temas de tesis en los últimos años en Francia.

5.- Trabajos de tesis realizados en los últimos años, así como el número de los títulos post-doctorales (HDR, o habilitación para dirigir las investigaciones), son claros ejemplos de esto. 
propósito (describiendo una situación, comprendiendo un fenómeno o explicando una manera de funcionar), enumerando todos los tipos de conocimientos y tomando sustento de una serie de experiencias metodológicas.

\section{Dos escuelas de pensamiento identificadas a menudo en la comunicación organizacional}

Existen dos paradigmas dominantes: el "positivismo", recurrente en la teoría de las organizaciones; y el "interpretativismo", que a menudo adopta una postura contra este, y excluye a un tercero, el denominado "constructivismo". En efecto, este último se ha alejado del vocabulario de las Ciencias de la Información y Comunicación (CIC). Si ha resurgido en los últimos años ${ }^{7}$, se ha producido dentro del ámbito de una polémica no resuelta que creemos escapa del ámbito del presente artículo. Este último paradigma también comparte un cierto número de hipótesis con el paradigma interpretativo.

En todo caso, si bien resulta necesario definir las características de estas dos perspectivas que permiten el estudio del fenómeno comunicacional, se debería evitar la confrontación entre ambas, ya que crearía una visión simplista que segregaría artificialmente la transmisión de la interpretación, dado que estas corrientes constantemente se entrelazan.

\subsection{El paradigma positivista}

Asociada con el paradigma positivista, también denominado la "versión balística de la comunicación" (Giordano, 1994), esta escuela de pensamiento considera la realidad social como un verdadero fenómeno ("principio ontológico"8), dotado de una existencia fuera del tema que observa y/o que lo hace ("principio de objetividad"), con una funcionalidad determinada y leyes para el éxito que son específicas para este ("principio del universo predeterminado") y que puede conducir a la solución óptima ("principio mínimo de acción u óptimo único").

Contra estos antecedentes, Giroux define la comunicación como "integrativa", lo que significa conductual con una visión colectivista de la organización. Como ejemplo, toma al gerente que reflexiona sobre los vínculos que lo unen a la organización y sobre su grado de integración dentro del colectivo organizacional.

6.- Revisar los trabajos de Berger y Luckmann; Chevalier, Delforce, Derville, Le Moigne, Piaget, Searle, Watzlavick... Esta enumeración muestra en qué medida esto implica una corriente que reúne opciones altamente diversificadas, que se presentan bajo los nombres de "construccionismo", "construccionalismo" o "constructivismo".

7.- Plasmados en varios números de la revista científica Questions de communication desde el año 2004.

8.- Aquí adoptamos los supuestos epistemológicos propuestos por Le Moigne (1990), para quien el positivismo considera la organización como una construcción ("principio del universo construido"), abarcando el sujeto que la intenta controlar o lo hace observándolo mediante la representación que hacen de este ("principios de representatibilidad y proyectividad"), teniendo una manera compleja de trabajar que no puede ser dividida en elementos simples e independientes y que solamente puede conducir a unas soluciones más o menos satisfactorias ("principio de acción inteligente"). 
Goldhaber (1986) considera que, con este enfoque, la comunicación organizacional es el proceso de la creación e intercambio de mensajes que, dentro de una red de relaciones interdependientes, tiene que adaptarse a la incertidumbre del ambiente. Sus trabajos conceptualizan la estructura organizacional distribuyendo los roles y las acciones de los individuos en propiedades, estableciendo niveles, departamentos y fronteras. La estructura organizacional se percibe como un "contenedor de entidades", de tal dimensión que las estructuras sociales existen antes que las acciones individuales.

Uno de los postulados básicos de la escuela funcionalista es la noción de "determinismo". Aquí encontramos el modelo telegráfico de comunicación” que retiene una propuesta de comunicación y se basa en la imposición de leyes y esquemas técnicos a los actores involucrados. Según esta perspectiva, los individuos son productos del ambiente y responden mecánicamente a los estímulos externos. Son esencialmente reactivos. La unidad de análisis retenida es la identidad organizacional con sus características sociales, psicológicas y económicas percibidas como entidades estáticas en vez de procesos sociales. La organización es una estructura concreta en la que surgen las actividades y la comunicación es una sustancia tangible que viaja hacia arriba, abajo o a los costados. Los mensajes son vistos como formas físicas que tienen posiciones espacio-temporales y existen independientemente de un emisor y un receptor.

De esta manera, para los investigadores funcionalistas, la esencia de la comunicación yace en la transmisión de mensajes y en el estudio de los efectos producidos por los canales de comunicación. Dentro de esta tendencia, más allá de los trabajos especializados en la identificación (George Cheney), el análisis de redes (Peter Monge; Noshir Contractor), la teoría de la estructuración (Robert McPhee) o la solución de conflictos (Cynthia Stohl), dos perspectivas parecen caracterizar a la escuela funcionalista (Axley, 1984; Daft, Langel, 1984; Jablin, 1987), hacia los enfoques mecánicos, por un lado, y a los enfoques institucionales, por el otro.

La perspectiva mecánica percibe la comunicación humana "como un proceso de transmisión" en el que el mensaje viaja a lo largo de un canal, de un punto al otro. Resalta el canal de comunicación como un medio de transmisión y también un vínculo entre la funciones de codificación y decodificación. Esta perspectiva transmite cuatro postulados básicos: la "cuasicausalidad", la "transitividad de las funciones de comunicación", la "conceptualización del materialismo" y el "reduccionismo". El primero se centra en el vínculo entre las condiciones previas y futuras: esta es una visión lineal del proceso comunicacional. El segundo considera que los conceptos comunicacionales están relacionados en una cadena de relaciones, mientras que la perspectiva mecánica trata la comunicación como una entidad material, lo que significa un mensaje que se convierte en una sustancia concreta con propiedades espacio-temporales. Finalmente, la comunicación puede dividirse en subunidades. Esta concepción reduccionista implica que los conceptos se comprenden mejor si la totalidad se divide en partes, mientras que se identifica todo a la vez y se mide esto último para revisar la cadena causal lineal que las une.

9.- Publicado en Théorie mathématique de la communication (Shannon y Weaver, 1948), este modelo designa un modelo de comunicación simple y lineal, reduciéndose meramente a la transmisión de un mensaje. 
La teoría institucional constituye el enfoque alternativo de la escuela funcionalista. La institucionalización a la que se refiere implica todos los procesos mediante los cuales las obligaciones o los problemas actuales asumen un estatus en el pensamiento y la acción social. Postula que las organizaciones cumplen con las expectativas del ambiente y se adaptan. A su vez, la organización es legitimada por el ambiente que le brinda sus recursos financieros y reconoce su estatus social.

Estos dos conceptos, a menudo, son adoptados por los profesionales y con frecuencia se encuentran en los manuales, especialmente aquellos que abarcan la comunicación interna.

\subsection{El paradigma interpretativista}

La escuela interpretativista considera a la sociedad como una construcción hecha con las experiencias subjetivas de sus miembros. Mediante su habilidad para comunicarse, los individuos son capaces de crear y construir su propia realidad social mediante sus palabras, símbolos y comportamientos. Las organizaciones son vistas entonces como procesos que se desarrollan a lo largo de los cambios en los patrones conductuales.

Asimismo, se interesa en la creación de significaciones compartidas por acciones y eventos comunes. El significado de las palabras y las acciones es interpretado de manera simbólica mediante la experiencia mutua en vez de recalar en la intención del remitente y en el filtro del receptor. El comportamiento se desarrolla a través de las interacciones sociales, cambia a medida que evoluciona el contexto social y forma una nueva entidad (Fischer, 1978).

Esta visión tiene implicancias en la manera en que se concibe la estructura organizacional. En efecto, las estructuras son tratadas como un conjunto de relaciones complejo y semiautónomo que toman a las interacciones como su origen. Los miembros de la organización utilizan sus acciones y sus interacciones para crear departamentos, niveles y procedimientos que tienen consecuencias directas en el comportamiento diario. El organigrama se vuelve simbólico ya que representa relaciones en procesos de cambio, pero también es estructural en tanto tiene un efecto en las acciones diarias de sus miembros. Dado que la escuela interpretativista trata a la organización como un conjunto de grupos con distintos intereses y metas divergentes, la visión que acarrea ya no es unitaria sino pluralista.

El método interpretativista encaja con una visión relativista del mundo: busca llegar a una comprensión profunda de un fenómeno en particular. Considerando que la organización es una construcción social de la realidad, el organizador se convierte, a su vez, en un proceso de comunicación. Según esa misma visión, la comunicación no es solamente otra actividad organizacional, sino que crea, legitima y recrea las estructuras sociales que forman el nodo de la organización (Hawes, 1974).

Este enfoque integra la comunicación en un sistema organizacional dinámico e interactivo en el que la realidad es construida conjuntamente por los actores y en el que los empleados de la compañía constituyen el cuerpo social, interactuando con la organización ("Escuela de Palo Alto"10). Los trabajos de la comunidad científica francesa, tanto para CIC como

10.- En 1959, Don Jackson fundó el Instituto de Investigación Mental en Palo Alto (California, Estados Unidos). Paul Watzlawick se unió a él en 1962. Con otros investigadores (Erving Goffman, Edward T. Hall, Gregory Bateson y Ray Birdwhistell) realizaron la investigación que cambiaría los paradigmas de la comprensión 
para las Ciencias Administrativas, se refieren principalmente a unas cuantas publicaciones, citadas abundantemente por Putnam y Weick. Debe recordarse que esta última investigación enfatiza el desempeño de los actores en la situación y en el lugar de la comunicación en las interacciones diarias. Considera la comunicación como una parte activa de un proceso de organización que contribuye con una permanente regeneración de las estructuras de la compañía y de los vínculos entre los individuos. El análisis de las situaciones ya no se realiza simplemente desde la reconstrucción ex post facto de los fenómenos, sino que se basa en las observaciones, resaltando la operación efectiva de la organización en tiempo real.

Podríamos referirnos a otros trabajos, incluyendo los enfoques postmodernos y críticos de Dennis y Mumby (2005). Sin embargo, mencionaremos sumariamente la tendencia discursiva de los enfoques críticos y foucaultianos; los enfoques conversacionales (Gail Fairhurst); y los enfoques narrativos (el "relato de historias", de Yannis Gabriel). Desde los trabajos altamente innovadores de James R. Taylor (Taylor y Van Every, 2000) sobre lo que se conoce como el enfoque "constitutivo" de la comunicación organizacional (Putnam, Nicotera, 2009), una serie de investigadores han iniciado diversos programas de investigación ambiciosos y fructíferos que buscan explorar el papel clave desempeñado por la comunicación en la constitución de las organizaciones (Cooren, 2000; Robichaud, Giroux y Taylor, 2004).

Según este enfoque, la comunicación se manifiesta esencialmente en dos modos: el "conversacional", se refiere hacia atrás a la dimensión relacionada a los eventos de cualquier interacción, que corresponde a los que Taylor y Van Every (2000) denominan la "zona de emergencia" de la realidad organizacional; mientras que el "textual" expresa la dimensión iterativa, repetitiva de cualquier intercambio, reflejando la "superficie" de la organización.

\subsection{Síntesis teórica}

En resumen, la perspectiva funcionalista considera la comunicación (junto con las líneas del "modelo telegráfico") como la transmisión de un transmisor a un receptor, mientras que la perspectiva interpretativista considera que la comunicación es construida por actores dotados de capacidades procesuales, cognitivas, afectivas y estratégicas durante los procesos de interacción involucrados en desarrollar significados. Aquí, la organización se convierte en un producto de comunicación.

En efecto, esto último sucede en el proceso de interacción entre los individuos y contribuye a construir el significado con la realidad. No es solamente una trasmisión única o aislada de un mensaje, sino una instancia para actualizar las representaciones individuales y colectivas que traen al juego significados elaborados previamente. Es el lugar donde se da el trabajo y se negocian las identidades de los actores. Mediante la comunicación, el colectivo humano se regula a sí mismo, se institucionaliza o, de lo contrario, se pone en cuestionamiento y transmuta. La comunicación, entonces, aparece como un proceso complejo en el que el significado de los mensajes no es algo dado antes de la interacción, sino una construcción en una situación marcada culturalmente que depende del progreso del proceso mismo en el tiempo y en el espacio.

de la comunicación sobre el llamado "modelo sistémico", por oposición al "modelo lineal", definiendo conceptos de "doble limitación", de "presentación de uno mismo" y "la dimensión escondida". 
Hay que admitir que la comunicación es también un producto de la organización (tanto comercial como no comercial ${ }^{11}$ ) cuando resulta de una opción deliberada para abordar una audiencia externa (Relaciones Públicas, creación de eventos, publicidad, marketing directo) o interna (boletín de la compañía, Intranet, reuniones) con o sin la ayuda de las $\mathrm{ICT}^{12}$; pero existe un continuo entre los dos que ahora se comprende claramente (Bouzon, 2004).

En nuestros días, la regulación busca promover la optimización de la gestión de los recursos humanos, naturales y financieros (internos y externos) para asegurar que se satisfagan las expectativas de las comunidades, especialmente haciendo que las empresas asuman sus responsabilidades con respecto a los bienes y servicios que producen y utilizan, llegando a dominar los "riesgos asociados", ya sean ambientales o sociales. Ahora, al reflexionar sobre la naturaleza de sus productos, las corporaciones se están volviendo cada vez más complejas en un ambiente crecientemente competitivo, en el que la variedad, la creatividad y la innovación están reemplazando gradualmente el criterio del precio y del volumen.

Por ese motivo, la comunicación alrededor del desarrollo sostenible (DS) constituye un problema emergente cuyo significado va bastante más allá de la especulación usual relacionada a la comunicación en momentos de crisis, dado que esta última solamente aborda una de sus facetas.

En ese sentido, los objetivos del DS hacen una referencia especial a la Responsabilidad Social Corporativa (RSE), dado que la tendencia del RSE no corresponde solamente a una "tendencia social". La RSE es un concepto mediante el cual las empresas abordan las preocupaciones sociales y ambientales (e incluso las gubernamentales) en sus actividades y en su interacción con sus stakeholders de manera voluntaria. De hecho, hasta ahora, ha habido pocas obligaciones, limitaciones o penalidades legislativas. Sin embargo, sería adecuado citar una ley relacionada a las nuevas regulaciones económicas (NRE) que exigen a las empresas incluir (en sus reportes anuales) una serie de informaciones sobre las consecuencias sociales y ambientales de sus actividades.

\section{Comunicación, incertidumbre y sostenibilidad}

Las expectativas de la sociedad, relacionadas a la seguridad de las personas y a la conservación del medio ambiente, están adquiriendo un papel más importante en el debate público. Esto se traduce en las respuestas que se deben brindar no sólo a las nuevas obligaciones legales, sino a las reglas para la seguridad de las personas y de la propiedad que, yendo más allá del conocido "principio preventivo", requieren un compromiso por parte de las empresas (incluyendo a las más pequeñas) para avanzar hacia la adopción de prácticas que hasta la fecha se habían limitado a las empresas más grandes.

11,- La teoría de las organizaciones define la organización como una unidad social que tiene metas y recursos compartidos, relacionándose tanto a estructuras comerciales como no comerciales, para acoger toda su diversidad (empresas, hospitales, sindicatos, asociaciones, administración, convenciones).

12.- En el presente artículo, las iniciales ICT se refieren a todos los medios digitales incluyendo Internet, extranet, correo electrónico y software de gestión integrado de la aplicación de planificación de recursos de la empresa, sino también los denominados "medios tradicionales". 
Definido como un tipo de desarrollo que asegura los por parte de las empresas (incluyendo a las más pequeñas) para avanzar hacia la recursos y satisface las necesidades del presente, sin comprometer el bienestar de las futuras generaciones, el DS todavía no se ha traducido en obligaciones limitantes impuestas a las empresas; estas asumen las preocupaciones sociales, ambientales e incluso de gobierno esencialmente de una manera voluntaria ${ }^{13}$. Pero esto implica nuevos deberes y un número mayor de actores, considerando la necesidad de difundir la información sobre la $\mathrm{RSE}^{14}$, especialmente en la elaboración de los informes.

Como resultado de ello, el desarrollo sostenible se ha convertido en un elemento esencial en la reputación de la empresa; todas ellas ahora deben pronunciarse sobre el DS, no solamente aquellas que operen en actividades "de riesgo". En ese sentido, se involucra al sector turismo y de transporte.

Los conceptos de RSE y DS son flexibles y están abiertos a interpretación, siendo moldeados por una gran cantidad de actores (Gond, 2006) que están aún emergiendo y están sujetos a cambio. En particular, se atribuyen a ellos cuatro principios para la acción: el principio "de responsabilidad", "de país contaminante", "de precaución" y "de la transparencia". Los dos últimos requieren, según nuestra opinión, una explicación adicional, dada su ambigüedad.

\subsection{Incertidumbre científica o "principio de precaución"}

Cualquiera que sea su campo de aplicación, el principio de precaución ${ }^{15}$, surgido en la década del ochenta, es un fenómeno social complejo que ha generado muchos trabajos académicos en los últimos años, debido a su variedad de usos y aplicaciones. Sin embargo, sufre de una extremada falta de precisión, especialmente debido a las dos concepciones que se disputan su campo.

La primera le atribuye la necesidad de garantizar un "riesgo cero", conjeturando sistemáticamente el escenario del peor caso, con la mejor sospecha de riesgo que conduce necesariamente a una moratoria o incluso a una retirada final, independientemente del aspecto del costo. En cambio, la segunda concepción solamente considera el principio de precaución ante la presencia de un riesgo probable que conduzca a un daño serio e irreversible. Recomienda la consideración sistemática de los costos económicos que se han de comparar con la ventaja esperada de las medidas preventivas.

Sin embargo, en nuestros días parece prevalecer una posición intermedia, donde se considera que la implementación del principio de precaución está subordinada al pronunciamiento de una hipótesis de riesgo científicamente creíble. Además, favorece la búsqueda para alcanzar una evaluación más precisa del riesgo, sin excluir la moratoria cuando se compruebe

13.- La RSE no puede ser completamente "voluntaria" en tanto que tales prácticas encajan en el marco del diálogo social, respondiendo a una cierta necesidad (Gendron, 2006).

14.- La RSE es la manera en que se desarrolla el desarrollo sostenible en la empresa. Se edifica en tres pilares: ambiental, social y económico.

15.- El principio de precaución (consagrado en la Ley francesa, 2/II/1995), estipula que "la ausencia de certezas, considerando en estado actual del conocimiento científico y técnico, no debe demorar la adopción de medidas efectivas y proporcionadas que buscan prevenir un riesgo con un daño serio e irreversible, a un costo económicamente aceptable". Sin embargo, hasta la fecha, ninguna legislación impone su aplicación directa. 
que es necesario, con un arbitraje final que se tiene que basar no solamente en los costos económicos sino en factores sociales, culturales y éticos (Viney, 2000).

Del mismo modo, la precaución implica brindar información sobre las características del producto o servicio; las condiciones para su uso o los recursos en el caso de un problema. Y no tan solo a lo que se refiere a los riesgos establecidos científicamente, sino que también aquellos que son previsibles o cuyas consecuencias se sospecha que se han comprobado completamente. En todo caso, cuando surge un riesgo, las organizaciones deben alertar inmediatamente al público, a través de los medios, para retirar el producto defectuoso a fin de ser revisado o reemplazado. Además, la aplicación del principio de precaución requiere de transparencia en cuanto a la naturaleza de los productos así como la introducción de la rastreabilidad sistemática. Esta transparencia abarca, en particular, el etiquetado de productos y servicios que permiten que se comparta balanceadamente las responsabilidades entre los ciudadanos informados, los proveedores y el Estado.

A los tres pilares del desarrollo sostenible, a nivel social (equitativo), económico (viable) y ambiental (habitable), se ha agregado el principio "de transparencia". Debido a las diversas presiones (y a la incidencia de consumidores y ciudadanos cada vez más exigentes) las organizaciones deben lidiar con dos nuevas formas de comunicación: la información pública y la transparencia. Esta orden se plasma en la RSE, en tanto está vinculada fuertemente con los principios de DS.

\subsection{Orden de comunicarse}

Para las principales empresas (que estuvieron dispuestas a responder) la comunicación relacionada a la $\mathrm{RSE}^{16}$ se presenta como estratégica: "El DS se ha convertido en un elemento esencial en las reputaciones de las empresas". "Todas las empresas deben comunicarse ahora sobre el DS y no solamente aquellas que se consideran que están "en riesgo"; "el DS se ha convertido en el denominador común de las muchas partes que se interconectan con la empresa." Pero los discursos pronunciados son convencionales y ninguno de nuestros interlocutores mencionó explícitamente el aspecto estratégico de la comunicación.

Sin embargo, los encuestados señalaron que la RSE requiere que se integren las preocupaciones sociales y ambientales en su política global de manera voluntaria. Incluso, si admiten que la legislación relacionada con ello es bastante irrestricta, los especialistas de la comunicación consideran que su ejercicio, que exige un respeto por el balance de intereses entre la gran cantidad de stakeholders (clientes, inversionistas, jóvenes graduados, empleados, proveedores, vecinos, ONG, las autoridades) con intereses muy variados, es “difícil”. Así, según las opiniones recogidas, no se puede separar de la estrategia de ventas y se traduce en una nueva política de comunicación, que se hace tanto más necesaria por la necesidad de preparar el informe anual obligatorio.

16.- La Responsabilidad Social Empresarial (RSE) se orienta hacia los empleados y, de manera más general, a todos los stakeholders que están relacionados con la empresa, los cuales pueden, a su vez, tener una influencia sobre su éxito. En Francia, la ley relacionada con las NRE (Nuevas Regulaciones Económicas) del año 2001, requiere que las empresas en la lista incluyan en el reporte anual una serie de informaciones relacionadas a las consecuencias sociales y ambientales de sus actividades. Además, el requerimiento de la RSE ha conducido a una provisión legal para las compañías en la lista, cuyo principio va más allá de la lógica voluntarista o de una simple recomendación. 
Cuando se les pregunta sobre este tema, los gerentes señalaron que la "comunicación" era un medio para respetar la legislación aplicable mediante la promoción de un tema capaz de reunir las preocupaciones de todos los stakeholders por igual, para converger con el mensaje correspondiente que se expone de manera expresa en todas las herramientas utilizadas.

Todas las empresas que respondieron insisten en la "imagen positiva" que esperan mostrar a sus clientes, accionistas y/o agencias crediticias ${ }^{17}$. También insisten en la necesidad de asegurar el control sobre el patrimonio de la información que se divulga dentro de su empresa, temiendo que su competencia la podría utilizar en detrimento suyo. Sin embargo, ninguno de nuestros interlocutores planteó las restricciones aplicables o el principio de precaución.

Más allá de la naturaleza universal o específica de los indicadores GRI, los especialistas de comunicación también consideran valorar el desempeño de sus empresas en los campos social y ambiental, con referencia a las características intrínsecas de sus respectivas organizaciones. Incluso, si la referencia a los diversos indicadores existentes se mantiene parcial (con indicadores variables y su uso supeditado) consideran que tal referencia alienta a las organizaciones a realzar sus programas ambientales y contribuir con la mejora continua del desempeño social y ambiental como se percibe de manera general.

En el análisis final, se eligen los indicadores de manera que la naturaleza distintiva de la organización pueda pasar a primer plano y se pueda adquirir una mayor legitimidad, yendo más allá de generar un informe de naturaleza casi obligatoria. ¿Los lineamientos GRI, que son extremadamente flexibles, permiten entonces que el lector informado implemente el benchmarking que desea el legislador y así comparar empresas del mismo sector, promoviendo de esta manera nuevas prácticas?

Los especialistas explican que los planes comunicacionales (versados en los esfuerzos realizados por la empresa en asuntos ambientales y sociales) pueden contribuir a aumentar el precio de las acciones de una empresa. El contenido del mensaje promovido parece corresponder al denominador menos común de todas las producciones de comunicación que están dirigidas a los objetivos externos.

¿Pero cuál es la situación cuando se trata de las personas en las compañías según lo que se considera expresamente en la legislación de la RSE? Las consideraciones aquí parecen posponerse en gran medida para una segunda etapa. De esta manera, solamente algunos expertos mencionaron cómo el informe (mediante el aumento de la conciencia sobre el desafío del DS) contribuye al orgullo de los empleados al señalar los resultados obtenidos y los esfuerzos realizados para contribuir con el desempeño social y ambiental de la empresa.

17.- Con 8 empresas (Accor, Air France-KLM, Crédit Agricole SA, le Groupe Danone, Lafarge, L'Oréal, Michelin and Saint-Gobain) pertenecientes a las cien más sostenibles, Francia alcanza el cuarto puesto en el "Ranking Global 100", cuyos resultados fueron publicados el miércoles 28 de enero de 2009 por el canal de medios Canadian Corporate Knights y la empresa consultora de inversión estadounidense especializada en administrar los riesgos "no tradicionales", Innovest Strategic Value Advisors. Global 100 es un proyecto que enumera a las cien empresas "más sostenibles". Estas son seleccionadas de entre las empresas que aparecen en la lista del índice mundial Morgan Stanley Capital International (MSCI), en base a los indicadores que determinan cómo una empresa confronta los riesgos y las oportunidades relacionadas al medio ambiente, los problemas sociales y el gobierno. 
Sin embargo, este aumento de la conciencia solamente se menciona en respuesta a nuestras preguntas sobre la consistencia entre los mensajes dados externa e internamente, haciendo una referencia sistemática a los valores y a la cultura corporativa como justificación. ¿Hubieran tocado el tema antes que los instemos a hacerlo? Algunos gerentes señalan que sí, empero, la comunicación es aprovechada por su habilidad para generar actividad. Así, es utilizada como un factor estratégico para institucionalizar lo que hasta entonces era simplemente una intención o una promesa.

Aquí encontramos uno de los temas desarrollados por algunos autores, como Libaert y Laville (2006: 228): "Al hablar abiertamente sobre su responsabilidad social y ambiental, la empresa se compromete a hacer que sus hechos sean acordes con sus palabras". No reflejaría, por ello, la realidad de las cosas, pero sí contribuiría a hacer que las cosas se conviertan en realidad. Esta hipótesis merece un desarrollo más detallado en un trabajo futuro.

Del mismo modo, en todas las entrevistas realizadas, quienes respondieron emplearon invariablemente los conceptos de RSE y DS, confundiéndolos o considerándolos sinónimos. Ya sea una acción consciente o no, parecería que la idea subyacente de la RSE está incluida en el DS. Pero los especialistas en comunicación consultados también parecen dudar y balancearse entre dos posiciones: sea el objetivo de un informe sobre DS (en términos de promover acciones "sostenibles" por parte de la organización) o la determinación de forjar un diálogo con los stakeholders.

Considerando el informe de la RSE, la estrategia de comunicación de la empresa también impone prácticas innovadoras, en las que los diversos socios de la empresa participan en la construcción conjunta de la estrategia de divulgación de la información. Así, el especialista en comunicación interactúa cada vez más con los diversos stakeholders, usuarios potenciales de la información generada (Perrini, 2005), pero también con otras empresas interesadas en la comunicación de este tipo de información (Jenkins y Yakovleva, 2006; Rowley, 1997) y expertos que ocupan este nuevo mercado del conocimiento (Gond, 2006), incluyendo el aspecto de benchmarking que mencionamos anteriormente.

Esto último plantea un desafío especial, ya que el informe sobre DS está dirigido a un público que no necesariamente está consciente de lo que realmente es el DS, incluso si esta difusión participa de un movimiento general de la sociedad en su conjunto hacia un objetivo común.

Así, a un nivel teórico, podemos considerar que las visiones positivistas y constructivistas mencionadas, que en un primer momento parecían opuestas entre sí o incluso contradictorias, demuestran ser complementarias. La organización y comunicación orientada a los turistas se construyen de manera conjunta y son realidades emergentes que forman una pareja inseparable. Además, el especialista en comunicación (al que se le confía el informe de RSE o de DS) parece ser un actor que interactúa con otros actores (tanto dentro como fuera de la organización) y a través de una "red de limitaciones" que dan forma a su lógica de acción según lo explica Gendron (2006). 


\section{Conclusiones}

Los conceptos sobre DS y RSE son vagos y maleables. Recién ahora están empezando a tomar forma. La referencia a los distintos indicadores existentes sigue siendo parcial, adoptando una frecuencia variable de divulgación que podría parecer más dependiente de las limitaciones internas de la empresa.

Los informes sobre DS deben integrarse a las herramientas de comunicación que están disponibles para las empresas. Los especialistas de RSE deben interactuar con los demás actores que forman parte de la construcción conjunta de tales conceptos y a implementar una práctica innovadora. Son ellos los llamados a definir, adaptar e interpretar los indicadores GRI para subrayar la naturaleza específica de la empresa. El éxito de esta actividad comunicacional yace entonces en su habilidad para tomar en cuenta las expectativas reales de los receptores del informe y hacer que su representación de DS evolucione.

La pregunta permanece en cuanto a los actos correspondientes que todavía son bastante aislados, resaltando el riesgo del marketing ético o el denominado "lavado verde" de las empresas.

Todo sucede como si los elementos de la RSE tuvieran la intención principal de dirigirse al público externo (mediante las RR. PP. o la publicidad), mientras que los valores asociados con el DS se utilizan principalmente para beneficiar a las audiencias dentro de la empresa. ¿Se da esto para preservar la coherencia y la compatibilidad de los mensajes, cuyas posibilidades de verificación son de naturalezas distintas, con empleados capaces de confrontar los mensajes mostrados con la práctica diaria? ¿O esto es simplemente fortuito? En cualquier caso, esta observación merece una investigación mayor.

Por ejemplo, con el desarrollo de Internet, las relaciones entre los stakeholders profesionales y los clientes se han visto sacudidas. La comunicación tiene que responder a la nueva morfología de los mercados, tornándose decisiva hacia las ventas de corto plazo mediante su presencia en la red.

Pero, ¿no se ha olvidado acaso el contenido de estos mensajes? En efecto, ofrece una imagen estereotipada que esquiva las características locales específicas, evadiendo cualquier choque entre las culturas particulares. Las producciones comunicacionales improvisan, exhibiendo una unidad visual superficial, una naturaleza "cosmética", hechas para encajar con otras expresiones de la empresa (logotipo y el sistema de identidad visual).

No obstante, junto con una arquitectura que integre los distintos canales que operan en sinergia entre sí, ¿no debería la comunicación ser un vehículo de mensajes concebidos para contribuir con la formación de una imagen de la marca a largo plazo? ¿No deberían considerarse los vectores para la comunicación y su contenido como un todo?

Ya sea que se considere como un discurso institucional o comercial y/o el fruto de la interacción permanente entre sus miembros o socios, la comunicación organizacional genera una especial atención entre los investigadores. Esto último toma tanto interés en su significado como en los recursos estratégicos implementados, los procesos 
comunicacionales y los fenómenos cognitivos y sociales adheridos a ellos. Al hacerlo, desarrollan modelos explicativos (cuya inspiración suele derivarse de otras disciplinas) que demuestran ser fuertemente dependientes de su propia cultura específica.

\section{Fuentes consultadas}

Acquier, A. y Gond J.P. (2006). "Les enjeux théoriques de la marchandisation de la responsabilité sociale de l'entreprise". Revue Internationale de gestion, 31(2), 83-91.

Antheaume N. y Teller R. (2001). "Quel regard vers d'autres formes de comptabilité: comptabilité sociétale et environnementale", pp. 85-99. En Dumontier P. y Teller R. (eds.). Faire de la recherche en comptabilité financière. París: Vuibert.

Bebbington, J.; Gray, R.; Thomson, I. y Walters, D. (1994). “Accountants' attitudes and environmentally-sensitive accounting". Accounting and Business Research, 24(94), 109-120.

Bouzon, A. (2004). La place de la communication dans la conception de systèmes à risques. París: L'Harmattan, collection Communication des organisations.

Callon, M.; Lascoumes, P. y Barthe, Y. (2001). Agir dans un monde incertain. Seuil.

Caron, M. A. y Turcotte, M. F. (2006). "Forces de transformation et d'inertie dans la divulgation de la performance d'entreprise : analyse de rapports de développement durable, Les cahiers de la Chaire d'information financière et organisationnelle". ESG-UQAM, núm. 2006-08.

Cormier, D., Gordon, I. M. y Magnan, M. (2004). “Corporate environmental disclosure: contrasting management's perceptions with reality". Journal of Business Ethics, 49(2), 143-165.

Cormier, D.; Magnan, M. y Feltham, G. (2005). "L'information comptable vue dans un perspective économique", pp. 239-253. En Colasse, B. (ed.). Les Grands Auteurs en Comptabilité, París: Éditions EMS.

Dogan, M. y Pahre, R. (1991). L’innovation dans les sciences sociales. París: PUF.

Euske N. A. y Roberts, K. H. (eds.) (1987). Evolving Perspectives in Organization Theory: Communication Implications, In Handbook of Organizational Communication: an interdisciplinary perspective. California: Sage Publications.

Fischer, B. A. (1978). Perspective on Human Communication. Nueva York: Macmillan.

Geertz, C. (1986). Savoir local, savoir global. Les lieux du savoir. París: PUF.

Gendron, C. (2006). Le développement durable comme compromis: la modernisation écologique de l'économie à l'ère de la mondialisation. PUQ.

Giordano, Y. (1994). "Business communication: Should managerial practices be reconsidered?" En Revue de gestion des ressources humaines, 13(4), 49-61.

Gond, J. P. (2006). Contribution à l'étude du concept de performance sociale de l'entreprise: fondements théoriques, construction sociale, impact financier [Tesis doctoral]. París: Université Toulouse 1 . 
Hawes L. C. (1974). "Social collectivities as Communication: Perspectives on Organization Behavior". En Quarterly Journal of Speech, 60, 497-502.

Henriques, I. y Sadorsky, P. (1999). "The relationship between environmental commitment and managerial perceptions of stakeholder importante". Academy of Management Journal, 42(1), 87-99.

Jenkins, H. M. y Yakovleva, N. (2006). "Corporate social responsibility in the mining industry: exploring trends in social and environmental disclosure". Journal of Cleaner Production, 14 (3-4), 271-284.

Jodelet, D. (ed.) (1999). Les représentations sociales. PUF.

Journe, B. (1997). "Positivisme et constructivisme dans la gestion de la sûreté et de la fiabilité des centrales nucléaires". Constructivisme et Sciences de gestion. Lille (octobre 1997).

Laville, E. (2002). L'entreprise verte. París: Village Mondial.

Le Moigne, J. L. (1990). Constructivist epistemologies of the organizational sciences. In Epistémologies et sciences de gestion. París: Economica.

Lerner, L. D. (1994). "Fryxell G.E., CEO stakeholder attitudes and corporate social activity in the Fortune 500". Business and Society, 33(1), 58-81.

Libaert, T. (2006). "Communication et développement durable: des relations ambiguës". Communication et langages, 150 (décembre 2006), pp. 127-133.

Lacoste, M. y Grosjean, M. (1999). Communication et intelligence collective. Le travail à l'hôpital. París: Presses Universitaires de France

May, S. y Mumby, D. K. (2005). Engaging Organizational Communication Theory and Research: Multiple Perspectives. California: Sage.

Perrini, F. (2005). "Building a European portrait of corporate social responsibility reporting". European Management Journal, 23(6), 611-627.

Redding, W.C.(1985). "Stumbling Toward Identity: The Emergence of Organizational Communication as a Field of Study". En McPhee, R. D. y Tompkins, P. K. Oganizational Communication: Traditional Themes and New Directions. Sage Annual Reviews of Communication Research. California: Sage Publications.

Roberts, R. W. (1992). "Determinants of corporate social responsibility disclosure: an application of stakeholder theory". Accounting, Organizations and Society, 17(6), 595-612.

Rowley, T. J. (1997). "Moving beyond dyadic ties: a network theory of stakeholder influences". Academy of Management Review, 22(4), 887-910.

Scardigli, V. (2001). Un anthropologue chez les automates. París: PUF.

Synnestvedt, T. (2001). "Debates over environmental information to stakeholders as a policy instrument". Eco-management and auditing, 8(3), 165-178.

Thayer, L. (1968). Communication and Communication Systems. Richard D. Irwin Inc.

Terssac, G. y Friedberg, E. (1996). “Coopération et conception”, pp. 1-22. Marseille: Octares. 
United Nations Publications (1987). Brundtland Report, World Commission on Environment and Development.

Viney, G. (2000). Le principe de précaution: Rapport au Premier Ministre. París: La documentation française.

Voos H. (1967). Organizational Communication: a Bibliography. New Brunswick: University Press. 